INNOVATIONS IN PRIMARY CARE

\title{
Scaling Integrated Behavioral Health Rapidly
}

Jobnny Stoeckle, $M D$

Amy Cunningbam, $\mathrm{PbD}, \mathrm{MPH}$

Christine Arenson, MD

Department of Family \& Community Medicine, Thomas Jefferson

University, Philadelphia, Pennsylvania

Ann Fam Med 2018;16:464. https://doi.org/10.1370/afm.2297.

\section{THE INNOVATION}

Integrated behavioral health (IBH), which is team-based care co-delivered by primary care and behavioral health clinicians, is being rapidly adopted by practices and health systems. The Center for Medicare and Medicaid's (CMS) Comprehensive Primary Care Plus (CPC+) program ${ }^{1}$ provided Jefferson Health with the opportunity to implement a system-wide IBH program. A key ingredient for success included cultivating buy-in at the c-suite (senior executive), management, and frontline levels.

\section{WHO \& WHERE}

Jefferson Health is a large academic health system with 60 primary care practices in $\mathrm{CPC}+$.

\section{HOW}

The CPC+ leadership team of primary care leaders and system administrators initially studied the opportunity to use CPC+ funds as seed money to hire behavioral health consultants to determine that it would meet strategic needs for mental health access. Billing models were created which predicted financial sustainability achievable prior to the end of the CPC+ funding period. These 2 findings created buy-in from senior leadership.

The interprofessional working group held biweekly planning meetings for 6 months prior to implementation. Creating an enterprise-wide IBH model was a top priority. Leading national models were examined and the Cherokee Health model, recently adopted by one of our campuses, was ultimately chosen with some local adaptations. ${ }^{2}$

An IBH leadership team composed of a Program Director, a Primary Care Liaison, and a Psychiatry Liaison was developed

Conflicts of interest: authors report none.

\section{Corresponding author}

Christine Arenson, MD

Department of Family \& Community Medicine

Thomas Jefferson University

1015 Walnut Street, Suite 401

Philadelphia, PA 19107

christine.arenson@jefferson.edu to ensure a successful, standardized implementation across diverse practice sites. This team, aided by the planning committee, was tasked with driving the launch of integrated behavioral health across the system.

Nineteen behavioral health consultant (BHC) positions were created and allocated across practices based upon Medicare patient volume. BHCs are licensed clinical social workers who manage the psychosocial aspects of acute and chronic diseases, apply behavioral principles to lifestyle issues, and provide consultation and comanagement for mental health disorders. Clinicians provide an in-person introduction ("warm handoff"). The $\mathrm{BHC}$ then delivers a brief intervention.

Clinicians were introduced to the model at strategic primary care meetings. Follow-up site visits, folded into routine population health management meetings, were performed to ensure proper adoption and to address distinct practice issues. Practices that demonstrated slower uptake were visited earlier to reinforce the model and address any concerns. We found the "80/20 rule" to hold: roughly $80 \%$ of clinicians were very positive in their reaction and uptake of the model; $20 \%$ required additional assistance.

Introductory training was provided for $\mathrm{BHCs}$, and ongoing meetings addressed updates and provided a feedback loop for issues. Group BHC supervision was established to address difficult cases and to quickly disseminate BHC best practices.

Finally, in order to demonstrate program process and outcome measures, tracking methods were standardized, and an IT workgroup began developing registries for caseload tracking and capabilities for targeted interventions. Additionally, Jefferson Health family medicine researchers will be assessing the level of primary care-behavioral health integration in Jefferson Health practices through regular administration of the Practice Integration Profile (PIP) ${ }^{3}$ and thorough evaluation of patient outcomes.

\section{LEARNING}

Scaling IBH rapidly requires strong backing from leadership and aligned buy-in from management and frontline workers. Significant interdisciplinary planning and coordination must be involved. IBH requires a significant shift in workplace culture, and this takes persistence and time. Employing rapid-cycle improvement with clear communication channels will optimize early adoption. Finally, these new models of care can lead to healthy integration of newly partnered health systems.

Key words, references, acknowledgments, and supplemental materials are available at http:I/www.AnnFamMed.orgl content/16/5/464/suppl/DC1. 